\title{
Effects of coordination and gender on prosocial behavior in 4-year-old Chinese children
}

\author{
Yingjia Wan ${ }^{1} \cdot$ Hong $\mathrm{Fu}^{1} \cdot$ Michael K. Tanenhaus ${ }^{1,2}$
}

Published online: 18 December 2018

(C) Psychonomic Society, Inc. 2018

\begin{abstract}
In a block-assembly task with 138, 4-year-old Chinese kindergarten children, tested in pairs, we manipulated whether finegrained coordination was required for accomplishing a shared goal with the same end product: building two adjoined towers with alternating levels of orange and green colored blocks to match a depicted model. In the coordination condition, each child had blocks of only one color and built the towers together. In the shared-goal-only condition, each child had both color blocks and built one of the towers, which they then adjoined. We predicted that children in the coordination condition would be more prosocial than children in the shared-goal-only condition. Studies with Western children typically find that girls are more generous than boys. However, we predicted the opposite pattern because Chinese culture emphasizes the importance of generosity more for males than females. Children in the coordination condition were more willing to help their partner complete an unrelated task and were more generous in sharing stickers with unknown children in a dictator game. These results demonstrate that level of coordination affects prosociality above and beyond having a shared goal, and are the first demonstration that prosocial effects of a collaborative task with children generalize beyond the participants to anonymous strangers. Boys shared more stickers with unknown children than girls, suggesting that gender differences in generosity are, in part, culturally conditioned.
\end{abstract}

Keywords Joint action - Collaboration · Coordination · Prosocial behavior · Cultural effects on cognition · Social cognition · Social development

From an early age, humans form a sense of joint commitment and "we" intentionality in joint activities (Michael, Sebanz, \& Knoblich, 2016; Tomasello \& Carpenter, 2007), which may lead them to be more helpful and generous during collaboration. Three-year-old children who have made a joint commitment are more likely to help their partner in time of need (Gräfenhain, Carpenter, \& Tomasello, 2013). When dividing up the results of collaborations, children tend to share resources fairly, even when given the opportunity to monopolize reward (Warneken, Lohse, Melis, \& Tomasello, 2010). Moreover, 3-year-olds divide rewards more equitably after

Hong Fu

njfuhong@139.com

1 School of Psychology, Nanjing Normal University, 122 Ninghai Road, Nanjing 210097, Jiangsu, China

2 Department of Brain and Cognitive Sciences, University of Rochester, Rochester, NY, USA collaborative efforts compared with working in parallel and no-task conditions (Hamann, Warneken, Greenberg, \& Tomasello, 2011).

Kirschner and Tomasello (2010) proposed that helpfulness and generosity generated by joint commitment and shared intentionality may extend beyond the original collaboration and influence young children's interaction in unrelated subsequent activities. This proposal has been examined by some adult studies: Some studies show significant prosocial effects (Reddish, Fischer, \& Bulbulia 2013); others report no such effects (Wolf, Launay, \& Dunbar, 2016). One possible explanation for these discrepant findings is that studies failing to find effects typically focus primarily on a shared goal, for example, a mutually desired end product or final state (e.g., a game that requires maximizing the total score of two players), whereas studies finding effects combine shared goals with more continuous coordinated behaviors, such as partner dancing (Fiebich \& Gallagher, 2013). More continuous coordination requires participants to pay attention to shared subgoals, during which they monitor and represent each other's 
tasks (Vesper, Butterfill, Knoblich, \& Sebanz, 2010). Moreover, immediate feedback for successful collaboration on each subgoal could reinforce prosocial tendencies (Reddish et al., 2013). Indeed, the constant representation of collective goals and close interaction with partners are characteristic of coordinated social behaviors that are known to increase mutual bonding and prosocial tendencies in both adults and children, such as joint music making (e.g., Kirschner \& Tomasello, 2010; Pearce, Launay, \& Dunbar, 2015) and synchrony (e.g., Hove \& Risen, 2009; Wiltermuth $\&$ Heath, 2009). Given these observations, we hypothesized that coordination plays a central role in determining the degree to which collaborative tasks will promote prosocial behavior.

Based on the adult literature on coordination together with the literature on 3-year-olds' and 4-year-olds' prosocial behavior, we hypothesized that coordination will generate prosocial behavior as early as 4 years, when children become capable of collaborating on problem-solving tasks (HolmesLonergan, 2003). We measured 4-year-old children's willingness to contribute to their partner's project and generosity following two problem-solving tasks. One task required only cooperating on a final goal. The other had the same end product and division of workload but required ongoing coordination. We predicted that coordination would increase prosocial behavior above and beyond having a shared goal.

We had two additional aims. First, we tested whether prosocial effects in a collaborative task would generalize beyond participants to anonymous strangers. Some proposed mechanisms for why coordinative activities enhance prosocial behavior, which include increased sense of unity (Wiltermuth \& Heath, 2009), perceived similarity (Valdesolo \& DeSteno, 2011), and greater mutual attentiveness (Macrae, Duffy, Miles, \& Lawrence, 2008), stress shared experience between coperformers. Thus, prosocial effects would be limited to participants. Indeed, several studies find that prosocial effects of coordinative activities are restricted to participants (Cirelli, Wan, \& Trainor, 2014; Tarr, Launay, Cohen, \& Dunbar, 2015; but cf. Cirelli, Wan, \& Trainor, 2016, who found that an infant being bounced in synchrony with an experimenter increases helpfulness only toward adults affiliated to the experimenter).

Alternatively, collaborative activities might promote prosocial behavior by increasing feelings of interdependence or priming cooperativeness (Over \& Carpenter, 2009). These mechanisms would suggest that effects would generalize beyond coparticipants. Some evidence with adult participants suggests that synchrony increases prosociality toward nonparticipants and even out-group members (Reddish, Bulbulia, \& Fischer, 2014; Reddish, Tong, Jong, Lanman, \& Whitehouse, 2016). If joint activities with shared intentionality are to foster the development of collaboration and socialization, it will be important to determine the conditions under which prosocial effects with child participants generalize. To the best of our knowledge, there is no existing evidence that prosocial effects of joint activities with children generalize to unknown children.

Second, we tested the hypothesis that Chinese boys would be more generous than girls. In Chinese culture, the importance of generosity with resources is stressed more for males than for females (Hou, Huang, \& Fang, 2017). Moreover, lack of generosity in women is more forgivable and sometimes even considered a virtue (Liao, 2012). To the extent that culture plays a role in shaping generosity and prosocial development (Benenson, Pascoe, \& Radmore, 2007), we would expect Chinese boys to be more generous than girls in sharing resources with unknown children. Greater generosity for boys would otherwise be surprising, given a large body of literature that suggests female children and adults are more helpful, cooperative, and generous than males (e.g., Eckel \& Grossman, 1998; Eisenberg \& Fabes, 1998; Kirschner \& Tomasello, 2010; but cf. Eagly \& Crowley, 1986).

\section{Method}

\section{Participants}

One hundred and thirty-eight children participated in the experiment. Children who did not follow instructions were excluded prior to analysis. Details are presented in the results. The final sample for the envelope task consisted of 129 children ( 69 boys and 60 girls, mean age $=4$ years and 6 months, range: $4.0-5.0$ years). For the star-arrangement task, the final sample included 116 children paired in same-sex dyads (58 boys and 58 girls, mean age $=4$ years and 6 months, range: $4.0-5.0$ years). Children were paired with a familiar partner of the same gender from the same kindergarten class. Parents and the school provided informed consent.

\section{Procedure}

\section{Manipulation phase}

Block-assembly task Pairs were randomly assigned to the coordination or the shared-goal-only condition. Pairs were presented with a model that looked like two adjoined towers (see Fig. 1). They were told that their task was to build two towers that looked exactly like the model. The experimenter built the bottom two levels of the tower to demonstrate the task.

In the coordination condition, one child was asked to build the parts that required the green blocks; the other used the orange blocks. In the shared-goal-only condition, one child was asked to build the tower on the left and the other the tower on the right. Assignment of color and side was random. Blocks were given to children in baskets. The experimenter 


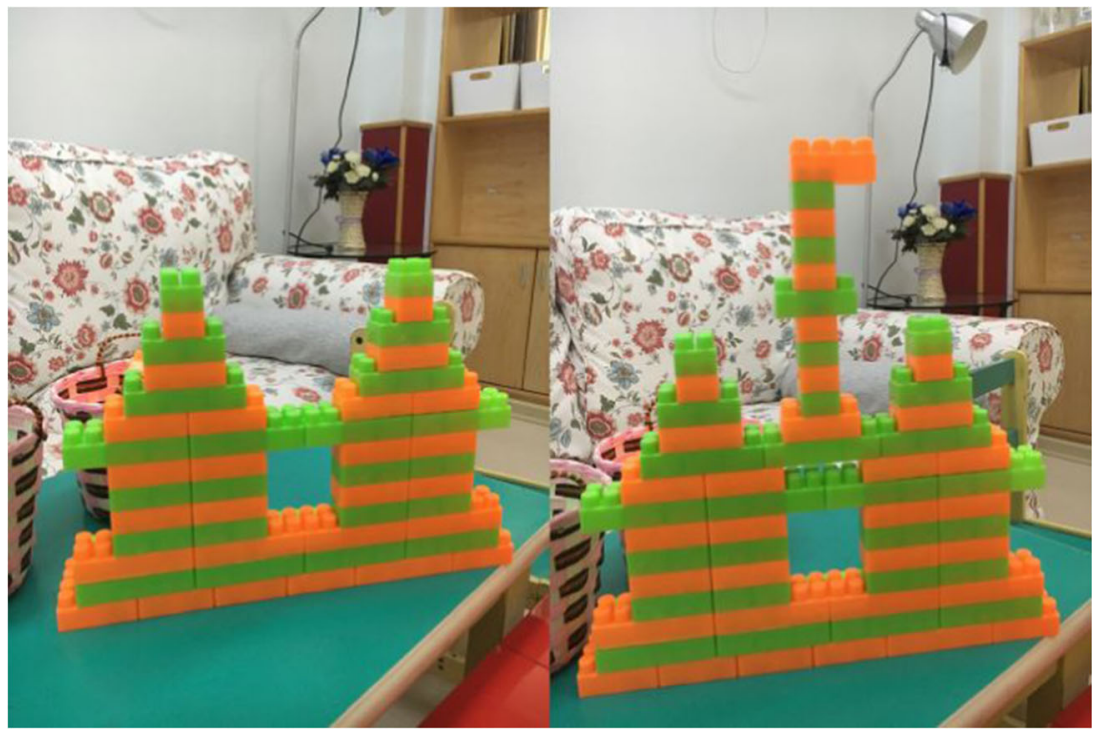

Fig. 1 Block-assembly models. The left panel shows the model for the first assembly task (building the towers); the right panel shows the model for the second assembly task (adding the flag)

and a research assistant were present to make sure that all the children followed the instructions. All children completed the task in approximately 5 minutes. Verbal interaction was minimal in both conditions, as children were each focusing on their own tasks.

The block-assembly task was followed by the first of two evaluation tasks: The star-arrangement task assessed prosocial behavior within the dyad; the envelope task assessed generosity to unknown children. After the first evaluation task, the pair added a flag to the towers to reinforce the manipulation. In the coordination condition, each child was responsible for one block color. In the shared-goal-only condition, one child built the bottom six levels of the flag; the other built the top six levels. Then they proceeded to the second evaluation task.

\section{Test phase}

\section{Star-arrangement task: Contributing to the partner's project} Each child was asked to finish a decoration pattern on a transparent card with stickers (see Fig. 2). The pattern was composed of one sun-shaped sticker and five star-shaped stickers of different colors. The experimenter first showed both children two finished models of the patterns that used different colors of stars and asked the children to name all the colors used in the patterns. After determining that both children could recognize the colors and count the numbers of stickers used, each child was given a model.

Each child was given a transparent card approximately $27 \mathrm{~cm} \times 9 \mathrm{~cm}$ with stickers attached to the left side of the card; the stickers given to each child are shown on the bottom panel below the models (see Fig. 2). The child was told to use the stickers on the left side to complete a decoration pattern on the right side that looked exactly like their model. The experimenter told the children there might be additional stickers, and they could do whatever they wanted with them, such as further decorating the pattern or putting the stickers on themselves, which was particularly popular among children in this kindergarten. The children were told that if they finished the pattern, they could keep the decoration card as a gift. All children showed great interest in this gift. After demonstrating with the sun-shaped sticker, the experimenter left the room and let the children finish the task.

The children sat parallel to each other and started the task. On all trials, Child B reported missing a star-shaped sticker of a particular color (blue for boys and pink for girls). Child A had a sticker of that color that was not needed to complete his or her Model A. The trial ended when both children had stopped working on their projects for 10 seconds.

\section{Envelope task: Sharing stickers with an anonymous child This} task took the form of a dictator game, adapted from Benenson et al. (2007).

The experimenter first asked the children if they would do her a favor. All children happily agreed. She then emptied a bag of 20 stickers in front of each child and asked the child to select 10 that he or she liked best. All children selected their stickers carefully and confirmed they liked their choices. The experimenter's subjective impression was that boys and girls were equally engaged and invested in selecting stickers. 


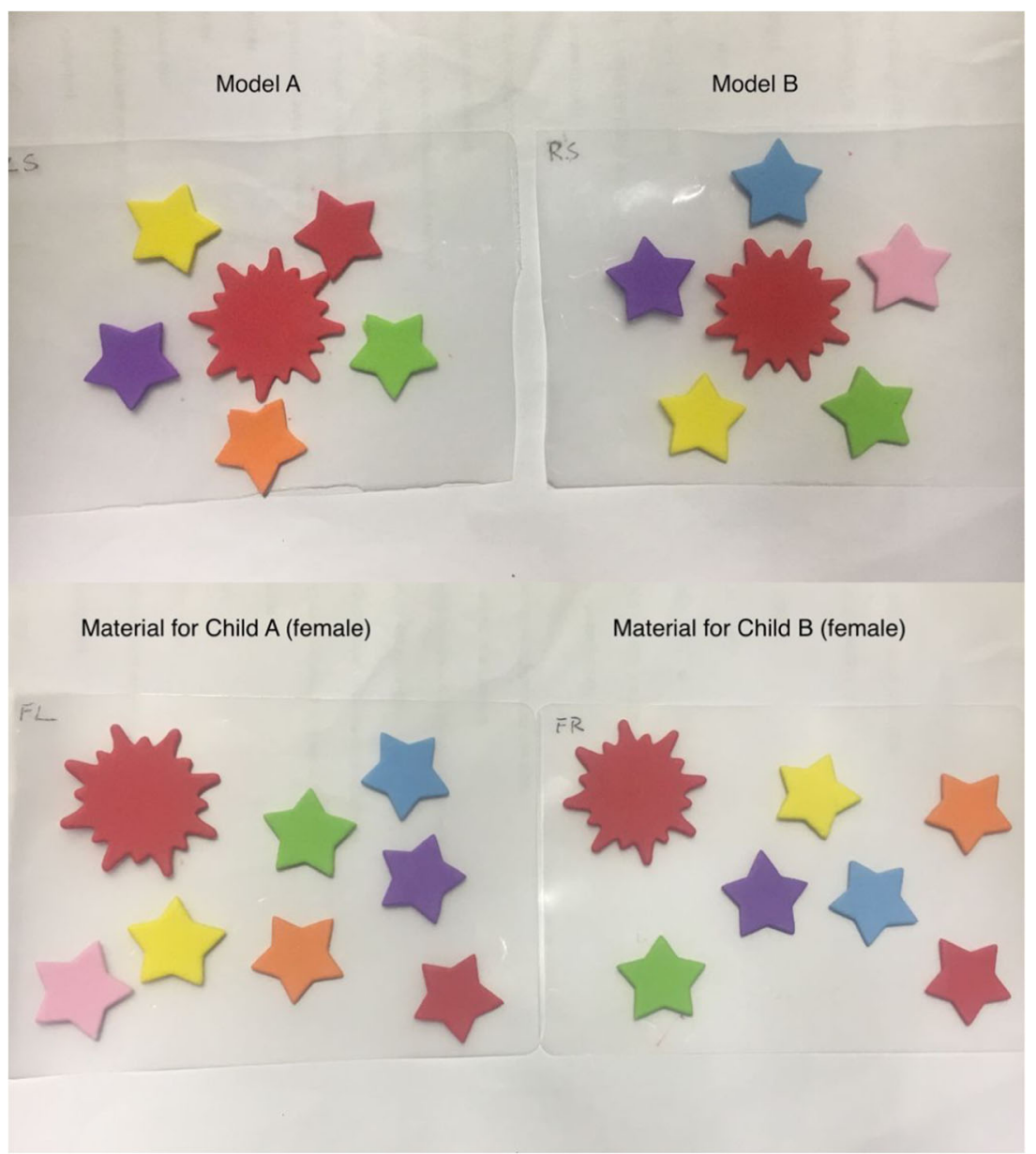

Fig. 2 Star-arrangement task materials (female). Top panel (Model A) was given to Child A: The five stars are yellow, red, green, orange, and purple. Top panel (Model B) was given to Child B: The five stars are purple, blue, pink, green, and yellow. Bottom panel shows the stickers given to Child A and Child B, respectively
The experimenter then told the children that she also planned to give stickers to children in other kindergarten classes. Because she did not have time to go to every class, she would need help dividing stickers. Children were told they could keep all 10 stickers for themselves or give some to children in other classes. Different classes in Chinese kindergartens rarely interact, so children are unlikely to know or feel affiliated with children from other classes. The experimenter gave each child two envelopes and drew a smiley face on one envelope. Children were told to put all of the stickers they wanted to keep in the envelope with the smiley face and to put any they wanted to share in the other envelope. The experimenter showed the children how to seal the envelopes and instructed them to seal both envelopes after dividing the stickers. She showed the children a basket of sealed envelopes and explained that the envelope without the smiley face would go into this basket and eventually be given to kids in other classes by another teacher. She emphasized that their choice was completely anonymous. She then told the children that she was separating them so that the other child would not know what choices they made. The two children were then brought to separate rooms. After explaining the procedure again, the experimenter left the room, leaving the child to divide the stickers. Children called out for the experimenter when they finished. ${ }^{1}$

The session lasted about 20 minutes. The order of the stararrangement task and the envelope task was counterbalanced across pairs and was balanced across trials. The experimenter (Y.W.) conducted the experiment with the help of a research assistant. All sessions were videotaped with a hidden camera.

\footnotetext{
${ }^{1}$ We conducted pilot testing with children from other kindergartens to make sure that 4 -year-olds would understand the tasks. Initially we gave the instructions to children after they were separated. Some children misunderstood the task, thinking they were donating stickers to their partner. This problem was eliminated when we revised the procedure to first give the instructions to the children together before separating the children, and then repeating the instruction.
} 


\section{Results and discussion}

\section{Star-arrangement task}

The video was coded by Y.W. and a research assistant blind to the hypotheses and coordination condition. Eleven of the 69 pairs were excluded prior to data analysis because one child did not follow the model. In the final sample, all 58 children who were missing a star reported it to the partner (e.g., "I'm missing a pink star!"). We coded the interaction between the dyad into one of four categories: (1) active giving (one child offered the spare sticker before the other child asked), (2) sharing after being asked (one child handed over the spare sticker after the other verbally requested), (3) taking (the other child took the sticker without getting permission), and (4) refusing to share (one child refused to share after the other child requested sharing or made an attempt to take the sticker). The four-category scale takes into account the actions of both children, thus increasing coding accuracy and allowing for post hoc analyses. Our hypothesis was about the effect of condition on willingness to share. Active giving and sharing after being asked both showed clear willingness to share, so we coded both as "willing to share" for the primary analysis. Interrater agreement $(\kappa)$ was 0.97 .

A logistic regression analysis found a significant effect of coordination on willingness to share $(\beta=1.62, z=2.80$, $p=.005,95 \%$ CI $[0.52,2.82])$. Children in the coordination condition were more likely to contribute to their partners' projects ( $77 \%$ of the trials) compared with those in the shared-goal-only condition (39\% of the trials). This result is not unexpected given the literature, but to the best of our knowledge, it is the first study to demonstrate coordination on a collaborative task increases prosocial behavior above and beyond having a shared goal.

Only the envelope task tests the hypothesis that boys will be more generous than girls. Nonetheless, we also examined gender differences in the star-arrangement task. There were no significant effects of gender on willingness to share and no interaction between the effects of gender and coordination. Some numerical trends for the subcategories were consistent with boys being more generous: Boys were less likely than girls to refuse to share (17\% vs. $31 \%$, Category 4$)$, whereas boys were more likely than girls to share when requested (67\% vs. $40 \%$, Category 2 and 4 combined). ${ }^{2}$

\footnotetext{
${ }^{2}$ Cross-culturally, boys are more aggressive than girls; we expect the same for Chinese children. M.K.T., who had not seen the tapes, suggested a post hoc analysis. Attempts to take the sticker without asking permission were coded as aggressive. Interrater agreement $(\mathrm{K})$ was 0.98 . A logistic regression yielded a significant effect of gender on aggression $(\beta=1.35, z=2.36, p=.018,95 \% \mathrm{CI}$ $[0.26,2.53])$ : Boys were more likely to attempt to take the sticker (55\% vs. $24 \%$ ), with no effect of condition.
}

\section{Envelope task}

Prior to data analysis, we excluded nine children who did not put all 10 stickers in one of the envelopes. For the remaining 129 children, we recorded the number of stickers placed in the "donate" envelope.

As shown in Table 1, coordination affected the number of stickers donated, with a mean of 3.17 in the coordination condition compared with 2.12 in the shared-goal-only condition. Boys donated more stickers than girls (3.20 vs. 2.10 ).

We evaluated the results using a regression analysis predicting the number of stickers donated, with coordination, gender, and task order as fixed effects. We included task order in the analysis because children who did the star-arrangement task first had an interaction that involved stickers prior to the envelope task. Including task order addresses the possibility that results in the envelope task could have been affected by prior experience with the stararrangement task. There were significant effects of both coordination $(\beta=1.07, t=3.03, p=.003,95 \%$ CI $[0.37$, $1.77])$ and gender $(\beta=1.12, t=3.17, p=.002,95 \% \mathrm{CI}$ $[0.42,1.81])$. There were no significant effects or interactions with order $(p s>0.5)$.

Figure 3 shows counts of children donating numbers of stickers by gender and condition. Because the numbers do not appear to be normally distributed, we used a Dirichlet process to infer the number of components of a Gaussian mixture model.

The best-fitting model, shown in Fig. 4, has two distributions (Distribution A: mean $=0.197, S D=0.614$, weight $=0.351 ;$ Distribution B: mean $=4.022, S D=1.255$, weight $=0.649$ ). By including 1.5 standard deviations around the means into the distribution, we coded donating zero and one sticker as falling into Distribution A and donating two or more stickers as falling into Distribution B.

A logistic regression predicted which distribution a child was assigned to, finding significant effects for coordination: $\beta=0.98, z=2.48, p=0.013,95 \%$ CI $[0.22,1.78]$ and gender: $\beta=1.21, z=3.03, p=0.002,95 \% \mathrm{CI}[0.44$, 2.01]. Children in the coordination condition were more likely to fall into Distribution B than were children in the shared-goal-only condition. Boys were more likely than girls to fall into Distribution B.

Table 1 Mean number of stickers donated for envelope task (standard deviations in parenthesis)

\begin{tabular}{llll}
\hline $\begin{array}{l}\text { Gender/ } \\
\text { coordination }\end{array}$ & Coordination & Shared goal only & Both conditions \\
\hline Female & $2.64(2.19)$ & $1.44(1.89)$ & $2.10(2.13)$ \\
Male & $3.65(1.81)$ & $2.69(2.10)$ & $3.20(2.00)$ \\
Both genders & $3.17(2.05)$ & $2.12(2.09)$ & \\
\hline
\end{tabular}




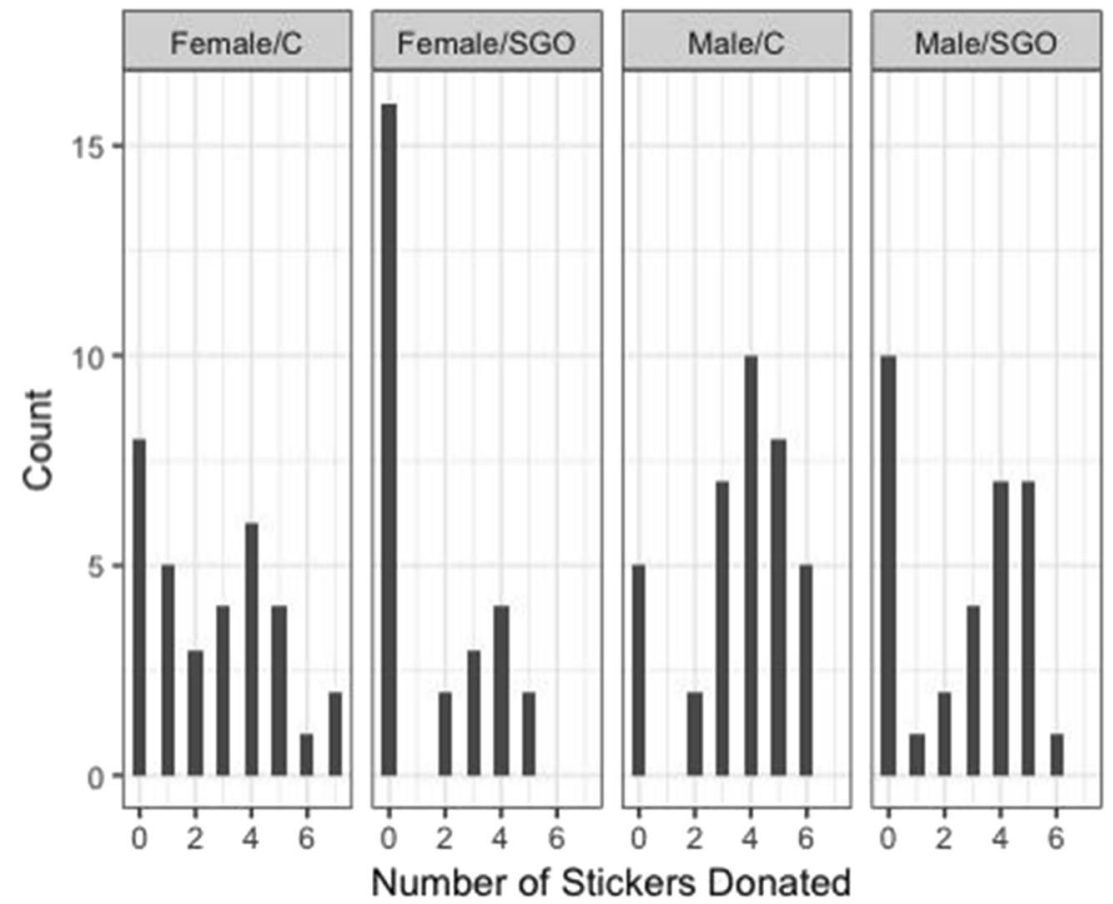

Fig. 3 Histogram of numbers of stickers donated. $\mathrm{C}=$ coordination condition; SGO = shared-goal-only condition

\section{Conclusion and implications}

The current study demonstrates that coordination increases 4year-olds' prosocial behavior above and beyond having a shared final goal within a dyad, and coordination also increases generosity to unknown children. These results suggest that joint activities involving fine-grained coordination could play an important role in the development of collaboration and socialization. In addition, Chinese boys were more generous than girls in sharing resources with unknown children, suggesting strong cultural effects on the development of gender differences in generosity. We consider the implications of each of these results in the remainder of the discussion.

\section{Coordination, shared goals, and prosocial behavior}

Collaborative activities and joint actions are presumed to involve shared goals, and having shared goals intuitively improves social relations (Tomasello, Carpenter, Call, Behne, $\&$ Moll, 2005). Nevertheless, the present study demonstrates that coordination on a collaborative task increases prosocial behavior above and beyond having a shared goal, which calls into question the prosocial effects of general collaborations, and leads to questions of what aspects of the interaction increase prosociality.

Joint attention increases social bonding and prosociality (Wolf et al., 2016). Children in the coordination condition most likely focused more on the same region than did children in the shared-goal-only condition. However, it is not clear why prosocial effects due to joint attention would generalize to generosity to unknown anonymous children. We are thus skeptical that the results in the envelope task can be primarily attributed to joint attention or any other partner-specific mechanism, including increased sense of unity (Wiltermuth \& Heath, 2009), perceived similarity (Valdesolo \& DeSteno, 2011), and greater mutual attentiveness (Macrae et al., 2008). Nonetheless, a promising direction for future research would be to examine the degree to which joint attention is involved in a collaborative activity. For instance, a finegrained measure of joint attention, such as eye movements, could be used to determine whether degree of joint attention

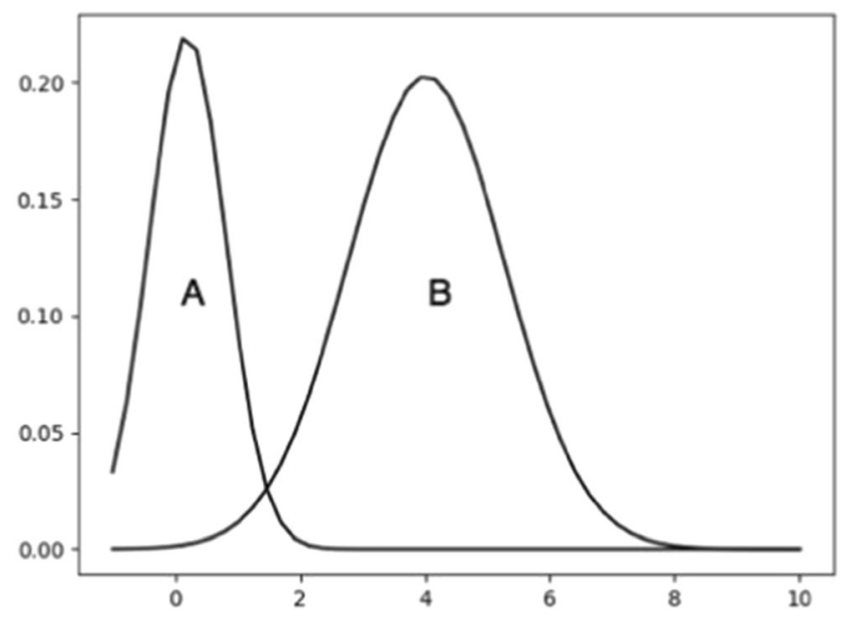

Fig. 4 Gaussian components of the Gaussian mixture model 
predicts prosocial behavior to partners and to unknown children in variations of the envelope task.

The generalization results seem more compatible with proposals in which coordinative tasks lead participants to experience others as cooperative/indispensable members of a social group (Reddish et al., 2013), whereas more discrete collaboration might introduce feeling of independence or even competition. In the coordination condition, children needed the blocks of the other child to be in place in order to start building a new row, whereas children in the shared-goal-only condition could finish their tasks on their own. It will be important for future research to manipulate and examine how different aspects of coordination (joint attention, mutual reinforcement, etc.) affect feeling of interdependence and prosociality, thus potentially adjudicating among alternative classes of explanation.

\section{Gender differences}

Our evidence that Chinese boys are more generous than girls in a dictator game is striking, given previous findings that girls are generally more generous than boys. Although we predicted that Chinese boys would be more generous than girls based on greater emphasis on generosity for males in Chinese culture, cross-cultural research will be necessary to establish a clearer causal relationship between socialization and gender differences. Importantly, cultural effects on gender differences in prosocial development might be more pervasive than previously appreciated, a topic that clearly merits further research.

Author note This study is supported by the Graduate Student Training Fund of Nanjing Normal University.

\section{Compliance with ethical standards}

Conflict of interest There is no conflict of interest in this paper.

Publisher's note Springer Nature remains neutral with regard to jurisdictional claims in published maps and institutional affiliations.

\section{References}

Benenson, J., Pascoe, J., \& Radmore, N. (2007). Children's altruistic behavior in the dictator game. Evolution and Human Behavior, 28(3), 168-175. doi:https://doi.org/10.1371/journal.pone.0080419

Cirelli, L., Wan, S., \& Trainor, L. (2014). Fourteen-month-old infants use interpersonal synchrony as a cue to direct helpfulness. Philosophical Transactions of the Royal Society B: Biological Sciences, 369(1658). doi:https://doi.org/10.1098/rstb.2013.0400

Cirelli, L., Wan, S., \& Trainor, L. (2016). Social effects of movement synchrony: Increased infant helpfulness only transfers to affiliates of synchronously moving partners. Infancy, 21(6), 807-821. doi: https://oi.org/10.1111/infa.12140
Eagly, A., \& Crowley, M. (1986). Gender and helping behavior: A metaanalytic review of the social psychological literature. Psychological Bulletin, 100(3), 283-308. doi:https://doi.org/10.1037/0033-2909. 100.3.283

Eckel, C., \& Grossman, P. (1998). Are women less selfish than men?: Evidence from dictator experiments. The Economic Journal, 108(448), 726-735. doi:https://doi.org/10.1111/1468-0297.00311

Eisenberg, N., \& Fabes, R. A. (1998). Prosocial development. In W. Damon \& N. Eisenberg (Vol. Ed.), Handbook of child psychology: Vol. 3. Social, emotional, and personality development (5th ed., pp. 701-778). New York, NY: Wiley. doi:https://doi.org/10.1002/ 9780470147658.chpsy0311

Fiebich, A., \& Gallagher, S. (2013). Joint attention in joint action. Philosophical Psychology, 26(4), 571-587. doi:https://doi.org/10. 1080/09515089.2012.690176

Gräfenhain, M., Carpenter, M., \& Tomasello, M. (2013). Three-yearolds' understanding of the consequences of joint commitments. PLOS ONE, 8(9), e73039. doi:https://doi.org/10.1371/journal. pone.0073039

Hamann, K., Warneken, F., Greenberg, J., \& Tomasello, M. (2011). Collaboration encourages equal sharing in children but not chimpanzees. Nature, 476, 328-331. doi:https://doi.org/10.1038/ nature 10278

Holmes-Lonergan, H. A. (2003). Preschool children's collaborative problem-solving interactions: The role of gender pair type and task. Sex Roles, 48, 505-517. doi:https://doi.org/10.1023/A: 1023523228455

Hou, J., Huang, J., \& Fang, X. (2017). The difference between implicit and explicit preferences for ideal partners. Studies of Psychology and Behavior, 15(4), 551-561.

Hove, M. J., \& Risen, J. L. (2009). It's all in the timing: Interpersonal synchrony increases affiliation. Social Cognition, 27, 949-960. doi: https://doi.org/10.1521/soco.2009.27.6.949

Kirschner, S., \& Tomasello, M. (2010). Joint music making promotes prosocial behavior in 4-year-old children. Evolution and Human Behavior, 31(5), 354-364. doi:https://doi.org/10.1016/j. evolhumbehav.2010.04.004

Liao, G. (2012). Examining linguistic gender difference from the perspective of adaption theory. Modern Communication, 2012(4), 58-59.

Macrae, C. N., Duffy, O. K., Miles, L. K., \& Lawrence, J. (2008). A case of hand waving: Action synchrony and person perception. Cognition, 109(1), 152-156. doi:https://doi.org/10.1016/j. cognition.2008.07.007

Michael, J., Sebanz, N., \& Knoblich, G. (2016). The sense of commitment: A minimal approach. Frontiers in Psychology, 6, 1968. doi: https://doi.org/10.3389/fpsyg.2015.01968

Over, H., \& Carpenter, M. (2009). Eighteen-month-old infants show increased helping following priming with affiliation. Psychological Science, 20(10), 1189-1193. doi:https://doi.org/10.1111/j.14679280.2009.02419.x

Pearce, E., Launay, J., \& Dunbar, R. I. (2015). The ice-breaker effect: Singing mediates fast social bonding. Royal Society Open Science, 2(10). doi:https://doi.org/10.1098/rsos.150221

Reddish, P., Bulbulia, J., \& Fischer, R. (2014). Does synchrony promote generalized prosociality? Religion, Brain and Behavior, 4(1), 3-19. doi:https://doi.org/10.1080/2153599X.2013.764545

Reddish, P., Fischer, R., \& Bulbulia, J. (2013). Let's dance together: Synchrony, shared intentionality and cooperation. PLOS ONE, 8(8), e71182. doi:https://doi.org/10.1371/journal.pone.0071182

Reddish, P., Tong, E., Jong, J., Lanman, J., \& Whitehouse, H. (2016). Collective synchrony increases prosociality towards non-performers and outgroup members. British Journal of Social Psychology, 55(4), 722-738. doi:https://doi.org/10.1111/bjso. 12165

Tarr, B., Launay, J., Cohen, E., \& Dunbar, R. I. (2015). Synchrony and exertion during dance independently raise pain threshold and 
encourage social bonding. Biology Letters, 84, 351. doi:https://oi. org/10.1098/rsbl.2015.0767

Tomasello, M., \& Carpenter, M. (2007). Shared intentionality. Developmental Science, 10(1), 121-125. doi:https://doi.org/10. $1111 / \mathrm{j} .1467-7687.2007 .00573 . x$

Tomasello, M., Carpenter, M., Call, J., Behne, T., \& Moll, H. (2005). Understanding and sharing intentions: The origins of cultural cognition. Behavioral and Brain Sciences, 28, 675-735. doi:https://doi.org/10.1017/S0140525X05000129

Valdesolo, P., \& DeSteno, D. (2011). Synchrony and the social tuning of compassion. Emotion, 11, 262-266. doi:https://doi. org/10.1037/a0021302
Vesper, C., Butterfill, S., Knoblich, G., \& Sebanz, N. (2010). A minimal architecture for joint action. Neural Networks, 23(8/9), 998-1003. doi:https://doi.org/10.1016/j.neunet.2010.06.002

Warneken, F., Lohse, K., Melis, A., \& Tomasello, M. (2010). Young children share the spoils after collaboration. Psychological Science, 22(2), 267-273. doi:https://doi.org/10.1177/0956797610395392

Wiltermuth, S. S., \& Heath, C. (2009). Synchrony and cooperation. Psychological Science, 20, 1-5. doi:https://doi.org/10.1111/j.14679280.2008.02253.x

Wolf, W., Launay, J., \& Dunbar, R. (2016). Joint attention, shared goals, and social bonding. British Journal of Psychology, 107(2), 322-337. doi:https://doi.org/10.1111/bjop.12144 